\title{
Inflammatory patterns in patients with chronic hepatitis C
}

\author{
Daniela Adriana Ion ${ }^{1 *}$, Mihaela A Rădulescu ${ }^{1,2}$, Victoria Aramă1,2, Raluca I Mihăilescu' , Cătălin Tilişcan ${ }^{1,2}$, \\ Violeta Molagic ${ }^{1}$, Cristina Popescu ${ }^{1,2}$, Anca-Ruxandra Negru', Viorica Poghirc', Daniela Adriana lon², \\ Adrian Streinu-Cercel ${ }^{1,2}$, Sorin Ștefan Aramă² \\ From The 9th Edition of the Scientific Days of the National Institute for Infectious Diseases Prof Dr Matei Bals \\ Bucharest, Romania. 23-25 October 2013
}

\section{Background}

In patients with chronic hepatitis $\mathrm{C}(\mathrm{CHC})$, liver fibrosis is a complex process, not completely understood, resulting from the activation of hepatic stellate cells and a proinflammatory status.

We aimed to evaluate the inflammation patterns in patients with $\mathrm{CHC}$ and the correlations between the inflammatory biomarkers and liver fibrosis.

\section{Methods}

We performed a cross-sectional study on patients with $\mathrm{CHC}$, in a tertiary-care hospital in November 2011 April 2012. We staged liver fibrosis using FibroMax (BioPredictive) and we used CobasTaqman (Roche) for HCV-RNA quantification. Patients were also evaluated by ALT, platelets, erythrocyte sedimentation rate (ESR), fibrinogen, C-reactive protein (CRP) and tumor necrosis factor alpha (TNFa) plasma levels. For TNFa quantification we used Kamiya Biomedical Company ELISA kits.

\section{Results}

We enrolled 76 patients with CHC (sex ratio M/F 0.6/1, median age 51 [44-58] years). According to the FibroMax evaluation, most of the patients had F0 fibrosis $(25 \%$, $\mathrm{n}=19)$, F2 fibrosis $(42 \%, \mathrm{n}=32)$ and F4 fibrosis $(16 \%, \mathrm{n}=12)$ respectively. The median HCV-RNA was 5 [5-5.75] $\log _{10}$. History of previous $\mathrm{HCV}$-therapy with pegIFN and ribavirin was recorded in $75 \%(n=57)$ of patients and half of them had a sustained virological response.

The inflammatory biomarkers displayed the following patterns: median ESR 12 [8-22.5] mm/h, median fibrinogen
295 [254-343] mg/dL, median CRP 0.55 [0.2-1.5] mg/L, median TNF alpha 6.38 [4.75-9.33] pg/mL.

Comparing the patients without fibrosis (F0) with the patients with fibrosis (F1-F4), we found a significant difference between TNFa values (patients with F0 - median TNFa 5.5 [4.0-7.1] pg/mL; patients with F1-F4 - median TNFa 7.3 [5.0-10.6]; $\mathrm{p}<0.001)$. In patients with fibrosis, we found a moderate correlation between TNFa values and fibrosis score, according to the FibroMax score (rho=0.4, $\mathrm{p}=0.002)$ and between TNFa values and ALT (rho $=0.43, \mathrm{p}=0.001$ ).

\section{Conclusion}

In patients with $\mathrm{CHC}$, liver fibrosis is correlated with the levels of TNFa, as a biomarker of systemic inflammation.

\section{Authors' details}

"National Institute for Infectious Diseases "Prof. Dr. Matei Balş", Bucharest, Romania. ${ }^{2}$ Carol Davila University of Medicine and Pharmacy, Bucharest, Romania.

Published: 16 December 2013

doi:10.1186/1471-2334-13-S1-O32

Cite this article as: Ion et al:: Inflammatory patterns in patients with chronic hepatitis C. BMC Infectious Diseases 2013 13(Suppl 1):032.

\footnotetext{
* Correspondence: danielaioana.mn@gmail.com

'National Institute for Infectious Diseases "Prof. Dr. Matei Balş", Bucharest,

Romania

Full list of author information is available at the end of the article
} 